\title{
Real-time Game Adaptation for Optimizing Player Satisfaction
}

\author{
Georgios N. Yannakakis, Member, IEEE, and John Hallam
}

\begin{abstract}
A methodology for optimizing player satisfaction in games on the 'Playware' physical interactive platform is demonstrated in this paper. Previously constructed artificial neural network user models, reported in the literature, map individual playing characteristics to reported entertainment preferences for augmented-reality game players. An adaptive mechanism then adjusts controllable game parameters in real-time in order to improve the entertainment value of the game for the player. The basic approach presented here applies gradient ascent to the user model to suggest the direction of parameter adjustment that leads toward games of higher entertainment value. A simple rule-set exploits the derivative information to adjust specific game parameters to augment the entertainment value. Those adjustments take place frequently during the game with interadjustment intervals that maintain the user model's accuracy. Performance of the adaptation mechanism is evaluated using a game survey experiment. Results indicate the efficacy and robustness of the mechanism in adapting the game according to a user's individual playing features and enhancing the gameplay experience. The limitations and the use of the methodology as an effective adaptive mechanism for entertainment capture and augmentation are discussed.
\end{abstract}

Keywords: User modeling, player satisfaction, real-time adaptation, augmented-reality games, neuro-evolution, gradient ascent.

\section{INTRODUCTION}

$\mathbf{C}$ OGNITIVE user models of playing experience promise significant potential for the design of digital interactive entertainment systems, such as screen-based computer or augmented-reality games. Quantitative modeling of entertainment or satisfaction - fun, player satisfaction and entertainment will be used interchangeably in this paper - as a class of user experiences may reveal game features or user features of play that relate to the level of satisfaction perceived by the user (player). That relationship can then be used to adjust digital entertainment systems according to individual user preferences to optimize player satisfaction in real-time [1]. In this paper, we analyze further the use of gradient ascent on a published user-preference model as an adaptive mechanism for achieving this in augmented-reality games. The children's game "Bug-Smasher" running on the 'Playware' [2] playground is used as the test-bed for the experiments presented here.

Entertainment models have already been constructed for the Bug-Smasher game [3], [4], based on quantitative measures

G. N. Yannakakis is with the Center for Computer Games Research, IT University of Copenhagen, Rued Langgaards Vej 7, DK-2300 Copenhagen S (e-mail: yannakakis@itu.dk); J. Hallam is with the Mærsk Mc-Kinney Møller Institute, University of Southern Denmark, Campusvej 55, DK-5230 Odense M, Denmark (e-mail: john@mmmi.sdu.dk). of Malone's intrinsic qualitative factors for engaging gameplay [5], namely challenge and curiosity. These models map game features, such as Malone's factors, and measures of the individual player's interaction with the game - player features - to a numerical value that represents the children's notion of 'fun'. Player features are derived from the statistics of the interaction of the player with the game platform. For Playware, this includes statistics derived from measurements of the time, location and foot pressure associated with interactions between the player and the platform. Neuro-evolution preference learning then uses these feature vectors to construct a function mapping the examined game and player features to the reported player satisfaction preferences such that preferred games receive higher function value. Feature selection techniques are applied to select the best-performing set of input features for the model.

Artificial neural network (ANN) models built in this way can achieve an average prediction accuracy, for children's preferences between Bug-Smasher variants, of $77.77 \%$ [4] (or $82.25 \%$ [6]) using four features (inputs): three player features (the player's average response time with the playground, the variance of the pressure force instances on the playground, the number of interactions with the playground) and the game feature of curiosity generated by the game opponents. Curiosity in the test-bed game investigated is measured using the spatial diversity of the opponents appearing in the game. The highest-performing ANN constructed - the same in both cited studies - achieves $90 \%$ prediction accuracy on expressed pairwise preferences. This particular model forms the basis of the adaptive controller discussed in this paper.

Following from the reported success with entertainment modeling in physical interactive games [4], a first attempt to optimize the entertainment value of those games in realtime was presented in [1]. A real-time adaptation mechanism, using gradient ascent on one of the models reported in [4] coupled with a simple rule-based controller, was implemented. Its performance was tested through a survey experiment in which children played variants of the Bug-Smasher game and reported which variants they preferred. Preliminary results [1] indicate a clear preference of children for the adaptive game versus a non-adaptive version. This paper extends the work of [1] by presenting a fuller analysis of the generated entertainment value and further discussion of the assumptions underlying the approach and its performance. The analysis indicates that in most cases the proposed mechanism does indeed adapt to the needs of the specific child in such a way as to increase significantly the child's satisfaction, and investigates those cases where this does not occur. These 
positive indications - for a simple gradient ascent mechanism applied to augmenting player satisfaction — suggest that future, smarter, implementations of adaptive learning in real-time may be even more effective for enhancing player experience.

The work reported here is novel in analyzing and discussing more thoroughly a demonstration [4] that a subjective model (a predictor of user preferences) of reported entertainment, grounded in statistical features obtained from child-game interaction, can be exploited to enhance player satisfaction in realtime by indicating suitable modulation of game parameters. The methodology proposed can be used to automate, in part, modern game development processes like quality assurance and testing but also to enrich human computer interaction through tailoring the interaction experience.

The paper is organized as follows. First, an extensive review of the literature on approaches for player satisfaction modeling and optimization is presented in Section II. Then, the basic steps of the methodology for constructing computational models of player satisfaction are outlined in Section III. Subsequently, an analysis of player satisfaction model accuracy with respect to gameplay time is presented (Section IV). Findings on the relationship between time and model accuracy drive the design (Section V) of the adaptation mechanism used. Section VI and Section VII present, respectively, the survey experiment designed to evaluate the adaptation mechanism and the statistical analysis of the entertainment values predicted by the model. The analysis shows that

- the entertainment value of the game is significantly increased when adaptation takes place, and that

- erroneous adjustments of the game's curiosity (and challenge) levels appear to influence the preference of children for adaptation.

The paper concludes with a discussion of the limitations and the suitability of the proposed methodology as a generic tool for optimizing experience in real-time.

\section{ENTERTAINMENT MODELING AND OPTIMIZATION}

We classify approaches for capturing the level of player satisfaction into qualitative and quantitative. The former category includes the specification of qualitative features and criteria that collectively contribute to engaging experiences in entertainment systems, derived from experimental psychology studies. The latter category includes studies quantifying reported qualitative criteria of entertainment and constructing models that quantify (in some appropriate way) the complicated mental state of satisfaction perceived while interacting with digital interactive systems [7]. Given that distinction, the approach for modeling player satisfaction presented in this paper is quantitative. Related work on methodologies for improving player satisfaction in real-time is presented at the end of this section.

\section{A. Qualitative Approaches}

Several researchers have been motivated to identify what is 'fun' in a game and what engages people when playing computer games. Psychological approaches include Malone's well-known principles of intrinsic qualitative factors for engaging game play [5], namely challenge, curiosity and fantasy. Challenge is defined as the uncertainty toward attaining a goal generated by the game mechanics; curiosity is the notion of what will happen next in the game; and fantasy is the ability of the game to show or evoke images of physical objects or social situations not actually present.

The theory of flow [8] is a composite of factors (e.g. loss of the feeling of self-consciousness, distorted sense of time) that indicate full immersion in or engagement with an experience (also called optimal experience). Incorporating the theory of flow [9] in computer games as a model for evaluating game design principles that yield enjoyable experience has been a focus of a few recent studies [10], [11].

A comprehensive review of the literature on qualitative approaches for modeling player enjoyment demonstrates a tendency for the proposed criteria to overlap with Malone's and Csikszentmihalyi's foundational concepts. An example of such an approach is Lazzaro's work on 'fun' clustering [12]. Lazzaro focuses on four entertainment factors derived from facial expressions and data obtained from game surveys on players: hard fun, easy fun, altered states and socialization. Koster's theory of fun [13], which is primarily inspired by Lazzaro's four factors, defines 'fun' as the act of mastering the game mentally and suggests that 'fun' is strongly dependent on learning while playing. Bateman and Boon [14] identify four different audience modes (play styles) for player-centered game design: conqueror, manager, wanderer and participant (relating to Lazzaro's factors). Specific game design principles for engaging each player style/type are also proposed in that study. An alternative approach to characterizing fun is presented in [15] where fun is composed of three dimensions: endurability, engagement and expectations.

A few indicative studies taken from the vast literature of the user and game experience field are considered in this section. The work of Pagulayan et al. [16], [17] provides an extensive outline of game testing methods for effective user-centered design of games that generate enjoyable experiences. Ijssellstein et al. [18] describe the challenge of adequately characterizing and measuring experiences associated with playing digital games and highlight the concepts of immersion [19] and flow [9] as potential candidates for evaluating gameplay. Ryan et al. [20] have considered human motivation of play in virtual worlds, attempting to relate it to player satisfaction. Their survey experiments demonstrate that perceived in-game autonomy and competence are associated with game enjoyment.

\section{B. Quantitative approaches}

All of the above qualitative approaches are based either on empirical observations of user studies or on linear correlations of measured user data (interaction and physiological data) with reported emotions derived from Likert scale [21] questionnaires. On the other hand, the quantitative approach presented in this paper builds on the qualitative modeling framework and takes cognitive modeling one step further by quantifying entertainment. Quantitative approaches formulate entertainment in terms of mathematical models which yield reliable numerical 
correlates for (or predictors of) 'fun', entertainment or excitement. Advances in quantitative player satisfaction modeling have established a growing community of researchers that investigate diverse methodologies for modeling and improving gameplay experience [7].

Iida's pioneering work on metrics of entertainment in board games was the first attempt at modeling 'fun' quantitatively: he introduced a general metric of entertainment for variants of chess games, based on average game length and possible moves [22]. A recent study by van Lankveld et al. [23] introduces the concept of incongruity [24] as a potential measure of entertainment. Incongruity is defined as the difference between the complexity of the game environment and the complexity of the mental model a human has of the game environment. Incongruity is measured through health points in a simple, turn-based, side-scrolling arcade game but no experiments to validate the study's hypotheses were performed [23]. This measure resembles the notion of challenge defined by Malone [5] and the factor of hard fun of Lazzaro [25].

Other work in the field of quantitative entertainment capture is based on the hypothesis that the player-opponent interaction - rather than the audiovisual features, the narrative or the genre of the game - is the property that contributes the majority of the quality features of entertainment in a computer game [26]. Given this fundamental assumption, a metric for measuring the real-time entertainment value of predator/prey games was designed, using quantitative estimators of game characteristics (such as challenge and curiosity) based on the player-game interaction. The developed metric was established as effective and reliable by validation against human judgement [27], [28]. Further experimental survey studies by Beume et al. [29], [30] demonstrate the generality of the proposed interest metric in different prey/predator game variants. A quantitative measure of flow derived from the subject's perceived gameplay duration is also introduced in those studies.

Earlier experiments by the authors [31] have shown that artificial neural networks and fuzzy neural networks can extract a better estimator of player satisfaction than a human-designed one, given appropriate estimators of the challenge and curiosity of the game and data on human players' preferences [32]. Those studies introduce the notion of comparative fun analysis for eliciting genuine subjective responses concerning complex notions like 'fun' or 'enjoyment' from test subjects. Using 2alternative forced choice survey questions - e.g. 'which of these two games was more fun to play?' - rather than a Likert scale [21], minimizes the assumptions made about subjects' notions of 'fun' and allows a fair comparison between answers from different subjects. The reliability of comparative fun analysis is shown through the accurate quantitative entertainment models constructed for both screen-based [32] and physical game test-beds [4].

In psychophysiological studies, games are equipped with affect recognizers which are able to identify correlations between physiological signals and the human notion of entertainment. Correlations between physiological signals and reported adult user experiences in computer games have been examined in [33], [34], [35], [36], [37], among others. This paper however, does not consider physiological signals as a basis for constructing cognitive models in games and furthermore focusses on games that incorporate physical activity [38].

\section{Optimizing Player Satisfaction}

Approaches towards optimizing player satisfaction can be classified as implicit and explicit. An approach is implicit when the objective function to be optimized is built on heuristics that are peripheral to player satisfaction. A typical example of such a function is the match between the challenge preferred by the player and that offered by the game opponent, optimization of which implies improved player satisfaction. On the other hand, explicit approaches optimize a function that explicitly maps to player satisfaction. The methodology used in this paper is explicit since it attempts to maximize a function value derived from a model of player satisfaction.

Within implicit approaches we generally meet use of machine learning techniques for adjusting a game's difficulty based on the assumption that challenge is the only factor that contributes to enjoyable gaming experiences. Given this assumption, difficulty adjustment implies entertainment augmentation. Such approaches include applications of reinforcement learning [39], genetic algorithms [40], probabilistic models [41] and dynamic scripting [42], [43] and neuro-evolution of augmented topologies [44]. Further implicit approaches [45] concentrate on generating visibly intelligent (i.e. believable) NPC behavior based on the assumption that believability alone contributes to player satisfaction. Unlike these approaches we explicitly focus on control of user satisfaction rather than game difficulty or believability.

User models have been constructed for the generation of adaptive interactive narrative systems that potentially optimize the experience of the user [46], [47], [48]. User preference modeling with respect to content (race track) creation in racing games has also shown a potential for enhancing the quality of playing experience in those games [49], [50]. However, human survey experiments that verify the belief that player satisfaction is in fact enhanced have not yet been reported in any of the aforementioned approaches.

Within the explicit methods for optimizing player satisfaction, robust adaptive learning mechanisms have been built to optimize the human-verified ad-hoc 'interest' (entertainment) metric for prey/predator games introduced in [26], [27]. Experiments showed that an on-line neuro-evolution mechanism [28], [51], [52], [53] and a player modeling technique through Bayesian learning [54] were each capable of maintaining or increasing the game's entertainment value while the game was being played. Effectiveness and robustness of the adaptive (neuro-evolution) learning mechanism in real-time has been evaluated via human survey experiments [27]. Furthermore, studies with the "Playware" [2] augmented-reality playground have shown that ad-hoc rule-based mechanisms [55] can successfully adapt a physical interactive game in real-time according to a user's individual play features and improve children's gameplay experience.

Following the theoretical principles reported by Yannakakis [56], this paper is primarily focused on the contributions of 
game opponents' behavior to the real-time entertainment value of the game. Other aspects of game design - e.g. game mechanics, game levels or game narrative - that may contribute to playing experience are not considered in this work (see [57] for a proof-of-concept experiment on automatic game design). Furthermore, instead of being based on empirical observations of children's entertainment, the work presented here uses quantitative entertainment models already constructed using experimental data obtained from a survey experiment with children playing with Playware playground [4]. An adaptive mechanism is proposed for augmenting children's satisfaction in real-time and an additional survey experiment validates its efficacy in the Bug-Smasher test-bed game.

\section{Constructing QuAntitative Entertainment (USER) MODELS}

The work described in this paper builds on quantitative user preference models whose construction and evaluation has been fully described in the literature. For completeness, a brief recapitulation of the key points follows. The reader is referred to [3] and [4] for further details of the test-bed used and the entertainment (user) modeling methodology employed.

The test-bed game used for the experiments presented here is called 'Bug-Smasher'. The game is developed on a $6 \times$ 6 square Playware [2] playground, comprising 36 tiles each incorporating processing power, communication, input (force pressure sensor) and output (light). The tile's dimensions are $21 \mathrm{~cm} \times 21 \mathrm{~cm} \times 4 \mathrm{~cm}$. During the game, different 'bugs' (colored lights) appear sequentially on the game surface and disappear again after a short period of time as a tile's light turns on and off respectively. The bug's position is picked randomly according to a predefined level of spatial diversity, measured by the entropy $(H)$ of the bug-visited tiles. The child's goal is to smash as many bugs as possible by stepping on the lighted tiles, thereby causing a force-sensor input to the tile. Pictures of the tile setup and snapshots of the Bug-Smasher game are presented in Section VI.

In [4], experimental data of platform-child interaction and children's entertainment preferences were acquired for BugSmasher. Three values ('Low', 'Average', and 'High') were defined for each of Malone's factors [5] of challenge and curiosity, giving 9 different game variants. Challenge was represented by the speed that the bugs appear and disappear in the game while curiosity was represented by their spatial diversity (tile-visit entropy). The former provides a notion of a goal whose attainment is uncertain and the latter effectively portrays a notion of unpredictability in the subsequent events of the game - the higher the entropy, the less predictable the next bug's location and therefore the higher the curiosity.

Seventy-two children aged from 8 to 10 years participated in the experiment reported in [4]. All participants were normalweighted $^{1}$, to minimize the effect of weight as a factor on physical interaction and playing experience. Children played two game variants for 90 seconds each; the two games differed in the levels of one or both entertainment factors of challenge and curiosity. For each completed pair of games, they reported their 'fun' preference using a 2-alternative forced choice protocol.

Pressed-tile events were recorded in real-time during play. From those data, nine personal (individual) player features were computed for each child. These included the fraction, $P$, of presented bugs successfully smashed (i.e. child's score); the number of interactions, $N_{I}$, with the game environment; the average, $E\left\{r_{t}\right\}$, and the variance, $\sigma^{2}\left\{r_{t}\right\}$, of the response times; the average, $E\left\{D_{b}\right\}$, and the variance, $\sigma^{2}\left\{D_{b}\right\}$, of the distance between the pressed tile and the bugs appearing on the game; the average, $E\{p\}$, and the variance, $\sigma^{2}\{p\}$, of the pressure recorded from the force pressure sensor; and the entropy, $H_{C}$, of the tiles that the child visited. The total number of game pairs played was 144; data from 7 game pairs was lost because of equipment failure, leaving a data set of 137 game pairs labeled with children's preference judgements.

To construct a quantitative user preference model from the game pair data, the Sequential Forward Selection (SFS) feature selection technique is applied to select that subset of a set of candidate individual player and game features that allows the best-performing artificial neural network model to be built. The SFS method is a bottom-up greedy search procedure in which one feature is added at a time to the current feature set. The feature to be added is selected from the set of remaining candidate features such that the new feature set generates the maximum value of the performance function over all candidates for addition [58].

The key assumption in model construction is that the entertainment value $y$ of a given game - which models the subject's internal response to playing the game, that is, how much 'fun' it is - is an unknown function of individual features which a machine learning mechanism can learn. The subject's expressed preferences constrain but do not specify the values of $y$ for individual games but we assume that the subject's expressed preferences are consistent.

The ANN representing the user preference model is constructed using a preference learning [59] approach in which a fully-connected ANN of fixed topology is evolved by a generational genetic algorithm (GA) [4] which uses a fitness function that measures the difference between the children's reported preferences of entertainment and the model output value $y$. The GA chromosome is a vector of ANN connection weights. To permit evaluation of the performance of a constructed ANN model, the available data is randomly divided into three subsets. Each of these is used as a validation data set in a run for which the other two-thirds of the data serve as the training set, resulting in three independent runs. The performance of an ANN model is measured through the average classification accuracy of the ANN in these three independent runs (a 3-fold cross-validation scheme). The result of this evaluation drives the feature selection process in its exploration of candidate feature subsets.

Experiments for finding the candidate feature subset yielding the highest ANN performance resulted [4] in a 3-fold cross-validation performance of $77.77 \%$ (average of $70 \%$, $73.33 \%$ and $90 \%$ ) when the ANN input (selected features from SFS) contains $E\left\{r_{t}\right\}, \sigma^{2}\{p\}, H$ and $N_{I}$. The binomial-

\footnotetext{
${ }^{1}$ Based on their body mass index lying between 18.5 and 25 .
} 
distributed probability of this performance to occur at random is $\mathbf{0 . 0 0 1 9}^{2}$. Further experiments reported in [6] achieve a higher 3-fold cross-validation accuracy of $82.25 \%$ (average of $76.66 \%, 80.00 \%$ and $90 \%$ ). In fact, the highest performing ANNs (90\%) derived in both these studies are identical; this ANN is the model used in this paper as the basis of the game controller. A further analysis of the feature subset $\left\{E\left\{r_{t}\right\}\right.$, $\left.\sigma^{2}\{p\}, N_{I}, H\right\}$ with the highest validation performances reveals that fast responding children $\left(E\left\{r_{t}\right\} \approx 0\right)$ tend to enjoy average and high curiosity values whereas slow children $\left(E\left\{r_{t}\right\} \approx 1\right)$ appear to prefer games that generate low curiosity levels [4].

\section{IV. 'FUN' DURING THE GAME}

At this point we have outlined how one can obtain quantitative models that predict children's preferences with high accuracy, given vectors of suitable feature values derived from a complete 90 second game. In other words, they quantify the 'fun' level of a game after it is finished. This is manifestly unsuitable for control of a game, since for that we need estimates of the degree to which the game is fun while it is in progress. To go further, we finesse this complication for now; we return to it in the discussion of this paper.

Suppose one could assume that the entertainment value of a game (the model output - denoted $y$ above) was constant throughout the game. Then one could use preferences expressed after the game to construct a model that evaluates the game using input features computed on sub-intervals of the game duration, and thereby obtain more frequent fun estimates. Alternatively, one could take a model trained using full-game data and explore how well it predicted wholegame preference based on input features computed from subintervals. Of these two possibilities we adopt the second, since input features can easily be scaled to compensate for a different duration over which they are calculated, and the model's output value for the sub-interval can be checked for consistency with the known whole-game user preference data.

This naturally raises the question of how long the subintervals should be: too short, and there may not be sufficient data to compute reliable feature values; but if long, the control points at which the satisfaction measure can influence the game will be too infrequent.

We therefore proceed as follows. Given three independent ANN models derived from whole game data - those reported in [4] and whose construction we outlined above - we calculate their average classification performance on the entire data set of 137 game pairs. Then the interaction data for each game is divided into two equal parts and all features are recalculated for these two 45 -second time windows. The three models are then re-tested on the entire new data set ( 2 times 137 data) assuming that the expressed whole-game entertainment preferences remain valid for both 45 -second segments. The model performance is re-calculated analogously for shorter intervals up to the point where a small time window is reached (e.g. 9 seconds). The goal is to determine the

\footnotetext{
${ }^{2}$ Underlined and bold p-values denote statistically significant effects; significance equals $5 \%$ in this paper.
}

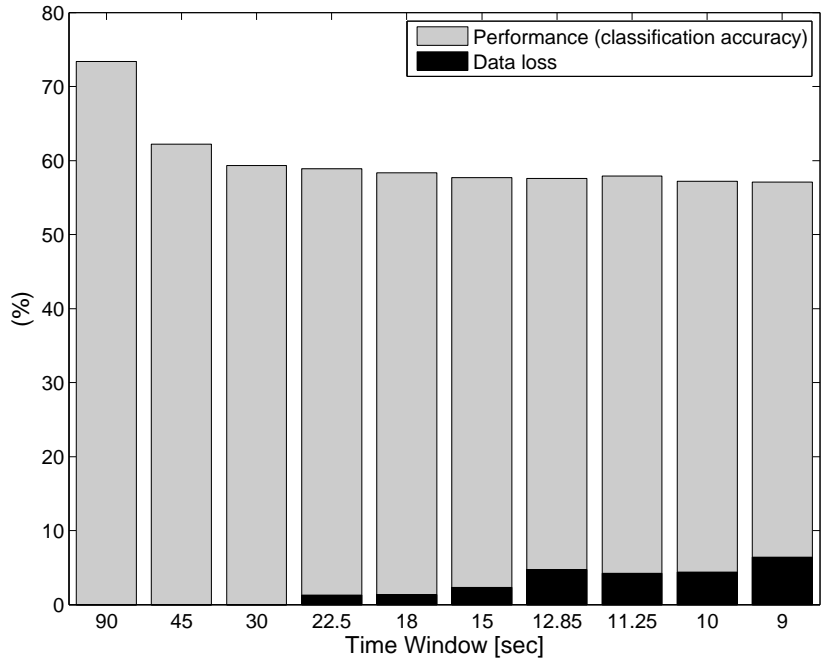

Fig. 1. ANN model performance and respective data loss percentage over different gameplay time windows. The two percentage values (columns) are independent in this illustration.

shortest time window for which the model can still predict reported entertainment with acceptable accuracy. That time window can then be used to set the frequency of any realtime adaptation mechanism applied.

Fig. 1 illustrates the classification performance of the ANN models with respect to the time window selected. The percentage of data lost for each time window is also shown in the figure: "data loss" occurs when there is no significant interaction between child and platform during the given interval, making it impossible to calculate the interaction-derived features. (Clearly, this becomes more likely as the interval decreases.) Results show clearly that performance is degraded by windowing the data, with an immediate decrease to $62.23 \%$ when the feature calculation interval is halved to 45 seconds. The performance stabilizes around $58 \%$ as the time interval is further reduced. However, data is lost when gameplay time windows become smaller than 30 seconds. This suggests that the minimum acceptable time window lies between 30 and 45 seconds: the models' performance, even though reduced, is still above $60.0 \%$ while data loss for these windows is zero.

Unfortunately, consecutive windows of 45 or 30 seconds mean infrequent control points: at most 2 in a game. We therefore investigate overlapping windows with time-shifted origins (TSOs). A TSO is the time interval between starts of successive feature computation windows. For instance, a 45second window with a 15 -second TSO generates the following four time intervals lying completely within the whole-game duration: $0-45,15-60,30-75$ and $45-90$ seconds. Table I shows the performance of the model and data loss for TSOs of $45,22.5$ and 15 seconds with a 45 -second feature computation window and TSOs of 30 and 15 seconds with a 30-second window.

ANN models evaluated on the 45:15 regime (45-second computation window starting every 15 seconds) yield the best performance of $64.53 \%$; moreover, no data is lost. This suggests that the 45:15 time scheme is the most appropriate of those tested here for real-time adaptation of the Bug-Smasher 
TABLE I

TIME-SHIFTED ORIGIN (TSO) IMPACT ON 45 AND 30-SECOND WINDOWS

\begin{tabular}{c|c||c|c|c}
\hline \hline Window & TSO & Data & Data Lost & Performance (\%) \\
\hline 45 & 45 & 274 & 0 & 62.23 \\
45 & 22.5 & 411 & 0 & 63.17 \\
45 & 15 & 548 & 0 & 64.53 \\
\hline \hline 30 & 30 & 407 & 4 & 59.34 \\
30 & 15 & 680 & 5 & 60.88 \\
\hline \hline
\end{tabular}

game. Furthermore, this implies that any game adaptation mechanism has 3 control points at which fun estimates are available, namely at 45 seconds and each 15 seconds thereafter - i.e. after 45,60 , and 75 seconds of play.

\section{REAL-TIME AdAPTATION MECHANism}

Given the ANN model of entertainment preferences built on individual gameplay features from Section III, and the fun estimation regime just described, the next logical question is how to use this model to improve children's gameplay experience in real-time. There are several ways of exploiting the model's built-in knowledge (discussed below in the discussion of this paper) and adapting Playware games for enhancing the level of entertainment. In this initial study we start with the simple gradient ascent mechanism presented in this section.

The idea behind real-time adaptation is to use the metric evaluation function (the ANN user preference model) directly to enhance the entertainment provided by the game. The key to this is the observation that the model represents a (differentiable) functional relationship of game features to entertainment value $y$. It is therefore possible in principle to infer what changes to game features will cause an increase in the entertainment value of the game, and to adjust game parameters to make those changes. Given the real-time average response time $\left(E\left\{r_{t}\right\}\right)$ of a child, the variance of his/her pressure forces on the tiles $\left(\sigma^{2}\{p\}\right)$ and the number of times he/she interacts with the environment $\left(N_{I}\right)$, the partial derivative of the model output $\vartheta y / \vartheta H$ can be used, for example, to appropriately adjust the level of entropy (curiosity) of the opponent $(H)$ so as to increase the entertainment value $y$.

$$
\vartheta y / \vartheta H=y(1-y) \sum_{i=1}^{M}\left\{o_{i}\left(1-o_{i}\right) w_{H, i}^{1} w_{i, 1}^{2}\right\}
$$

where $M$ is the number of hidden neurons; $o_{i}$ is the output of the $i$-th hidden neuron of the ANN model; $w_{H, i}^{1}$ is the connection weight between the $H$ input and the $i$-th hidden neuron and $w_{i, 1}^{2}$ is the connection weight between the $i$-th hidden neuron and the output neuron of the ANN model.

Since the $\vartheta y / \vartheta H$ value indicates the change in entertainment for a small change in the curiosity level, one could use gradient ascent to attempt to improve entertainment with such a model. Note, however, that this derivative tells only in which direction to change $H$ and not by how much, and further that this approach is only possible for game features since they are directly controllable.
Previous studies [4] have shown that the number of interactions and the average response time are features correlated linearly with entertainment preferences. These features are determined by the individual player, but are strongly influenced by the game speed (challenge) — which is a controllable game feature. The effects of game speed on the number of interactions ( $\mathrm{p}$-value $=\underline{\mathbf{7 . 6 3} \cdot \mathbf{1 0}^{-\mathbf{7}}}$ ) and average response time $\left(p\right.$-value $=\underline{1.51 \cdot 10^{-4}}$ ) are statistically significant. Therefore, in addition to curiosity level adjustment, the game speed $(s)$ is adapted in real-time, by a simple set of rules given below, to influence these two player features.

Analogously to equation (1), the partial derivatives $\vartheta y / \vartheta N_{I}$ and $\vartheta y / \vartheta E\left\{r_{t}\right\}$ are calculated. The game's speed is altered if those values have different signs: higher speed for positive $\vartheta y / \vartheta N_{I}$ and negative $\vartheta y / \vartheta E\left\{r_{t}\right\}$; lower speed for negative $\vartheta y / \vartheta N_{I}$ and positive $\vartheta y / \vartheta E\left\{r_{t}\right\}$. Table II presents the complete set of rules used for adjusting the curiosity and challenge levels of the game using a 45:15 second time window regime in a 90 second game - that is, adjustments occur on the $45^{\text {th }}$, the $60^{\text {th }}$ and the $75^{\text {th }}$ second. Adjustments are implemented by altering the state ('Low', 'Average', 'High') of the internal controls (challenge, curiosity) by one level up $(+)$ or down (-). Note that when $|\vartheta y / \vartheta H|<\epsilon$ (third row of Table II), curiosity is randomly increased or decreased with equal probability.

TABLE II

ADAPTATION MECHANISM RULES. $\epsilon$ EQUALS 0.1 IN THIS PAPER.

\begin{tabular}{c|c}
\hline \hline Condition & Action \\
\hline \hline$\vartheta y / \vartheta H>\epsilon$ & $H+$ \\
\hline$\vartheta y / \vartheta H<-\epsilon$ & $H-$ \\
\hline$|\vartheta y / \vartheta H| \leq \epsilon$ & $H+$ or $H-$ \\
\hline \hline$\left(\vartheta y / \vartheta E\left\{r_{t}\right\}<-\epsilon\right)$ AND $\left(\vartheta y / \vartheta N_{I}>\epsilon\right)$ & $s+$ \\
\hline$\left(\vartheta y / \vartheta E\left\{r_{t}\right\}>\epsilon\right)$ AND $\left(\vartheta y / \vartheta N_{I}<-\epsilon\right)$ & $s-$ \\
\hline \hline
\end{tabular}

\section{ADAPTATION EXPERIMENT}

The adaptive mechanism was tested with Bug-Smasher. Two variants of the game were constructed: the static and the adaptive. The bugs' speed $(s)$ and the entropy of bugvisited tiles $(H)$ for the static game were adjusted to the average values of the three different levels ('Low', 'Average', and 'High') of challenge and curiosity respectively used in the Bug-Smasher experiments [4]. (A reviewer of this work made the good suggestion that the average preferred levels of challenge and curiosity would have made a better control game than that used. The computed difference between average $s$ and average preferred $s(0.065 \mathrm{sec})$ and the corresponding difference in average $H(0.0248)$ do not appear large with respect to the interval these values lie within - $[0,1.5]$ and $[0,1]$ respectively — and suggest that the static game is, nevertheless, a valid control.) The adaptive game was initialized with the same values for speed and spatial diversity as the static game but the challenge and curiosity levels were 
adjusted during play, according to measured player interaction, using the adaptation rules presented in Table II three times at 45, 60 and 75 seconds.

For the adaptation experiment, we asked 24 naive ${ }^{3}$ normalweighted children (13 boys and 11 girls) aged 8 to 10 years to play 4 games each on the Playware platform. The set of 4 games played comprised 2 games of static and 2 games of adaptive Bug-Smasher in all combinations. Thus, the number of children participating in the experiment is $4 \cdot C_{2}^{4}=24$, this being four times the required number of all combinations of 2 out of 4 games. Subjects play games in pairs and each time a pair of games is finished, the child is asked to choose using the 4 alternative forced choice (4-AFC) protocol below:

- the first [second] game was more 'fun' (see [15] for terminology used in experiments with children) than the second [first] game ( $c f$. 2-alternative forced choice)

- both games were equally 'fun' or

- neither of the two games was 'fun.'

Note that children are not interviewed but are asked to fill in a comparison questionnaire, minimizing the interviewing effects reported in [33]. Children complete a questionnaire after games 2,3, and 4, resulting in three fun comparisons (expressed preferences) between games $1-2,2-3$, and 3-4, for each child to report. That provides a total of 72 (24 children times 3 comparisons) "fun" comparisons. The 4-AFC protocol above is used since it offers several advantages for subjective entertainment capture: it minimizes the assumptions made about children's notions of "fun" and allows a fair comparison between the answers of different children, while also making explicit the "no preference" cases concealed by 2 -AFC. The 4AFC protocol provides the same preference information as the 2-AFC used in previous experiments [4], [60] for any machine learning process applied to construct entertainment models.

As an example of a playing behavior, Fig. 2 depicts videocaptured photographs of the initial and final seconds of an adaptive game played by a subject that expressed a preference for the adaptive over the static game. This participant played the static game second and the specific adaptive game first. As seen in Fig. 2(a) the game starts with low levels of curiosity corresponding to bugs that mainly appear at the right hand side of the topology. As the game proceeds the curiosity level is increased $(\mathrm{H}+)$ which results in bugs appearing in a less predictable manner and in more tile positions (see Fig. 2(b)). Curiosity adjustments follow the rules presented in Table II and result in an increased entertainment value (compared to the entertainment value generated from the static game).

\section{A. Adaptive vs. Static Bug-Smasher}

Given the experimental protocol there are 50 out of 72 'fun' comparisons between the static $(S)$ and the adaptive $(A)$ Bug-Smasher. Table III illustrates the number of preference instances for each of the four alternative choices of 4-AFC. The number of instances for the $(A=S)$ alternative (20) and

${ }^{3}$ To check whether the children had any previous experience of the experimental procedure or the game, which for good experimental protocol they should not have, they were asked if they had seen the game before. None had.

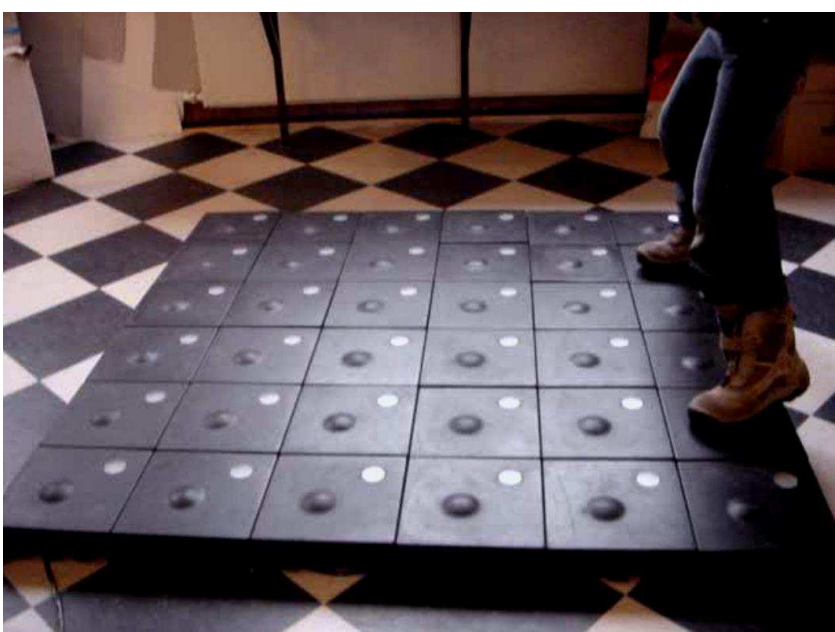

(a) Snapshot at the $5^{\text {th }}$ second of the adaptive game

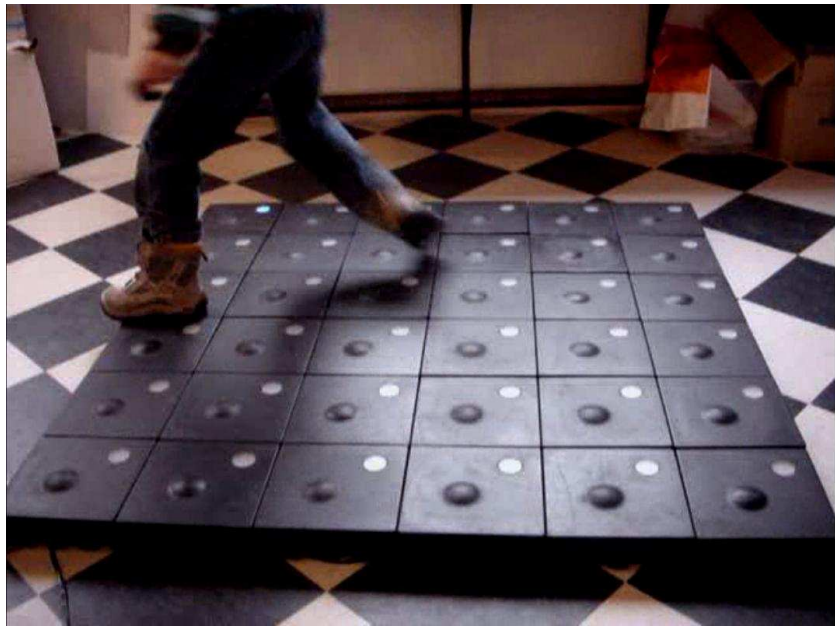

(b) Snapshot at the $80^{\text {th }}$ second of the adaptive game

Fig. 2. Subject no. 6 playing the adaptive Bug-Smasher game that adjusts the level of curiosity $(H)$ and challenge $(s)$ in real-time. The adaptation rules during this game are: $\mathrm{H}-(45 \mathrm{sec}), \mathrm{H}+(60 \mathrm{sec})$ and $\mathrm{H}+(75$ sec). Note that subject no. 6 preferred the adaptive over the static game he played. The ANN input recorded at the end of the static game is $N_{I}=60$, $E\left\{r_{t}\right\}=1.433 \mathrm{sec}, \sigma^{2}\{p\}=1.7 \cdot 10^{4}$ and $H=0.496$; the equivalent ANN input at the end of the adaptive game is $N_{I}=42, E\left\{r_{t}\right\}=2.032 \mathrm{sec}$ $\sigma^{2}\{p\}=22.0 \cdot 10^{4}, H=0.675$. The static and the adaptive game generate entertainment values of 0.058 and 0.7311 respectively. Video available at www. itu. dk/ yannakakis/playware.html.

the 'neither was fun' alternative (0) indicate respectively the difficulty for the respective children of distinguishing between the two games and that both games offered an enjoyable experience to all children participating in the experiment. The number of preference instances of Table III result in a percentage of $60 \%$ and $76 \%$ for respectively higher, and higher or equal, preference for the adaptive game versus the static game. The binomial-distributed probability of these two percentages to occur at random is 0.1002 and $\underline{\mathbf{1 . 5 9} \cdot \mathbf{1 0}^{-\mathbf{4}}}$ respectively. Even though not statistically significant, the percentage of children who prefer the adaptive game encourages belief in the effectiveness of the simple adaptation mechanism proposed here. 
TABLE III

INSTANCES OF SUBJECT PREFERENCES BETWEEN THE ADAPTIVE $(A)$ AND THE STATIC $(S)$ GAME

\begin{tabular}{l|c}
\hline \hline Choice & Instances \\
\hline Adaptive is more fun $(A \succ S)$ & 18 \\
Static is more fun $(A \prec S)$ & 12 \\
Equally fun $(A=S)$ & 20 \\
Neither was fun & 0 \\
\hline Total & 50 \\
\hline \hline
\end{tabular}

\section{Statistical Analysis of the Entertainment} VALUE

Next, we investigate whether the adaptation mechanism has a positive impact on the entertainment value of the game. For this purpose, we calculate the entertainment values $y$ of the aforementioned 50 static $\left(y_{s}\right)$ and adaptive $\left(y_{a}\right)$ full (90second) game pairs played during the experiment, given the ANN model and its input features. The assumption made here is that each user of the system playing the same game variant (i.e. static game) twice will generate a similar entertainment value through the ANN model. This assumption is supported by a $t$-test for means of paired samples which demonstrates no significant difference in the entertainment value between the static games played by the same child ( $t=0.7325$, $P(T \leq t)=0.2412)$.

In 13 out of 50 game pair instances the absolute difference $\left|y_{a}-y_{s}\right|$ is lower than 0.05 and is not considered for further investigation since the significant difference level is set to $5 \%$ of the $y$ interval. In 28 out of the remaining 37 games $(75.67 \%)$, adaptation improved the entertainment value regardless of the static game's generated entertainment value $y_{s}$. The binomial distributed probability of this performance to occur at random is $\mathbf{0 . 0 0 1 2}$ demonstrating the efficacy of the adaptation mechanism in increasing the entertainment value of the game significantly. Fig. 3 summarizes all above-mentioned observations in a $\left(y_{a}-y_{s}\right)$ stem plot for all 50 game pair instances.

A clear observation derived from Fig. 3 is that the adaptive mechanism increases the game's $y$ value independently of subjects' entertainment preference. Another observation is that the number of game pair instances with corresponding insignificant change of $y\left(\left|y_{a}-y_{s}\right|<0.05\right)$ is higher in the class of $A=S$ preferences (7 instances) than the class of $A \succ S$ (4 instances) and $A \prec S$ (2 instances) preferences. This observation suggests that the generated $y$ values have an effect in the $A=S$ expressed preference situation, and might explain the children's difficulty in choosing one of the two games played.

\section{A. Adaptation Maladjustments}

There are many factors that might have affected the entertainment preference of children with respect to real-time adaptation. Our hypothesis is that erroneous $H$ and $s$ adjustments by the adaptation mechanism, that lead to lower $y$ values, is the

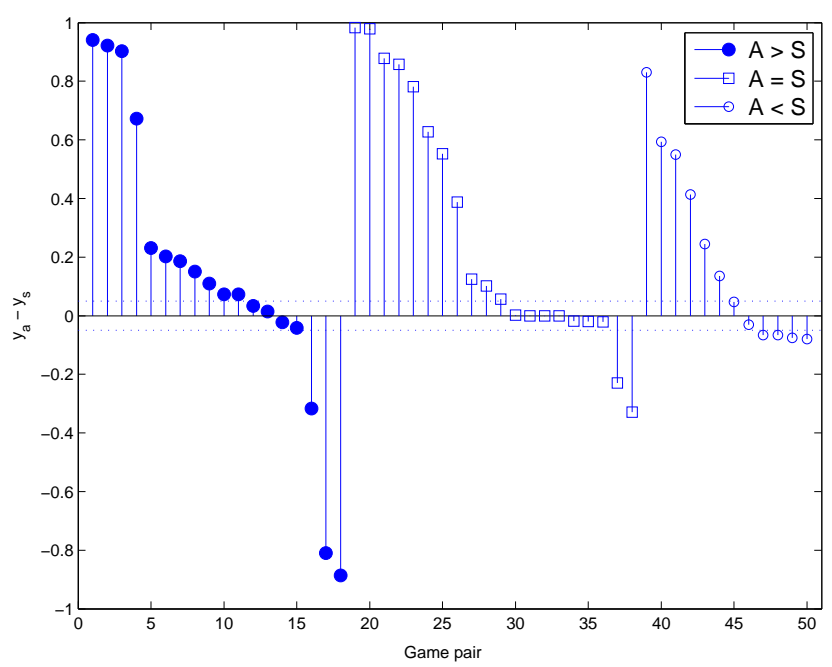

Fig. 3. Stem plot of $y$ difference between the adaptive and the static game $\left(y_{a}-y_{s}\right)$. Filled circles $\left(\bullet^{\prime}\right)$ represent preference of the adaptive over the static game $(A \succ B)$; circles ('o') represent preference of the static over the adaptive game $(A \prec B)$; squares represent equal preference $(A=B)$. The area between the dotted lines indicates the $5 \%$ significance level of $y$. Game pairs in each preference class are sorted in descending order of the $y_{a}-y_{s}$ value.

key factor correlated to entertainment preferences. To examine this, we compute the entertainment values predicted by the model at the $45^{t h}, 60^{t h}$ and $75^{\text {th }}$ second of each adaptive game played. We investigate 150 parameter adjustments in total since 3 game parameter adjustments occur during each of the 50 adaptive games. Only $18.67 \%$ (28 out of 150) of those parameter adjustments lead to a lower $y$ value being computed the next time an adjustment is made (15 seconds later). On the other hand, increased $y$ value is observed in 57 of those parameter adjustments (as mentioned before, absolute differences lower than 0.05 are excluded from further investigation). Even though the proportion of maladjustments is quite low, most of these erroneous real-time adaptation decisions result in very low $y$ values from which the adaptive system cannot easily recover before the end of the game. Decrease of $y$ is mainly caused by maladjustments of the tile visits entropy, $H$, since there are only 3 maladjustments of the speed parameter, $s$, in the dataset leading to lower $y$ values.

Further insight is possible by investigating the correlation of those maladjustments with the entertainment preferences expressed. Fig. 4 illustrates $y$ difference values due to game parameter adjustments for three classes of preferences: a) adaptive game is preferred to static game $(A \succ S$, see Fig. 4(a)); b) static game is preferred to adaptive game ( $A \prec S$, see Fig. 4(b)) and c) adaptive and static game are equally 'fun' ( $A=S$, see Fig. 4(c)). Table IV summarizes the percentages of total maladjustments, game instances with at least one maladjustment and maladjustments leading to lower entertainment values, for each of the three classes of preference. There is a great difference in the proportion of maladjusted games between the two classes of opposite preference: $A \succ S(33.33 \%)$ and $A \prec S(83.33 \%)$. Moreover, while only $16.66 \%$ of those maladjustments result in lower $y$ values in the class of children that preferred the adaptation 
game, $40 \%$ of those maladjustments have a negative impact on the $y$ value in the class of children that preferred the static game. This suggests that maladjustments during the game affect children's preference for the adaptive game. The correlation coefficient between entertainment preferences (the 20 instances where $A=S$ are excluded) and maladjusted adaptive games equals 0.4910 with a corresponding p-value of $\underline{\mathbf{0 . 0 0 5 9}}$ demonstrating the significant impact of adaptation maladjustments on the entertainment preference of children.

An additional experimental effect seen in Fig. 4 is that adjustments at the $45^{\text {th }}$ second have, on average, a lower impact on the $y$ value than adjustments at the $60^{\text {th }}$ and the $75^{\text {th }}$ second of the game. This effect is confirmed by the absolute $y$ difference values at those times: $0.162,0.291$ and 0.3155 respectively.

In addition to the maladjusted game factor, the impact of the random $H$ decision (see Table II) on children's entertainment preferences was also examined. However, results obtained do not demonstrate any significant effect.

TABLE IV

PERCENTAGES OF TOTAL MALADJUSTMENTS, GAME INSTANCES WITH AT LEAST ONE MALADJUSTMENT AND MALADJUSTMENTS LEADING TO

$y_{a}<y_{s}$ OVER THE THREE CLASSES OF PREFERENCE: A) ADAPTIVE IS PREFERRED TO STATIC GAME $(A \succ S)$; B) ADAPTIVE AND STATIC GAMES HAVE EQUAL PREFERENCE $(A=S)$; C) STATIC IS PREFERRED TO ADAPTIVE GAME $(A \prec S)$;

\begin{tabular}{l||c|c|c}
\hline \hline & $A \succ S$ & $A=S$ & $A \prec S$ \\
\hline Total maladjustments & 14.81 & 15.00 & 30.55 \\
Game instances & 33.33 & 35.00 & 83.33 \\
Maladjustments leading to $y_{a}<y_{s}$ & 16.66 & 14.28 & 40.00 \\
\hline \hline
\end{tabular}

\section{DISCUSSION}

The main assumption underlying this use of entertainment models for game control is that an entertainment preference expressed at the end of a game is valid for the game as a whole. This rather dubious assumption stands up quite well in the work reported above, but in general one might wish to obtain within-game preference data directly rather than estimate it from end-of-game data. Obtaining entertainment preferences during play is certainly a protocol an experiment designer may follow. However, such a protocol is likely to be intrusive to the user, augmenting the 'noise' present in the expressed preferences and affecting the validity of the data collected. Ideal conditions for effective data collection, proposed by Picard et al. [61], recommend that subjects should not be questioned during the task under examination (playing the game) since this interferes with the user's playing behavior. Thus, questioning during play was not adopted since it was judged a priori to be too intrusive and, a posteriori, unnecessary.

It is generally a hard task to acquire proper and 'clean' data for building cognitive models using machine learning. To the best of the authors' knowledge there is no study investigating the impact of time intervals between questions on expressed responses. Monitoring physiological signals that correlate to sympathetic arousal (e.g galvanic skin response) could provide

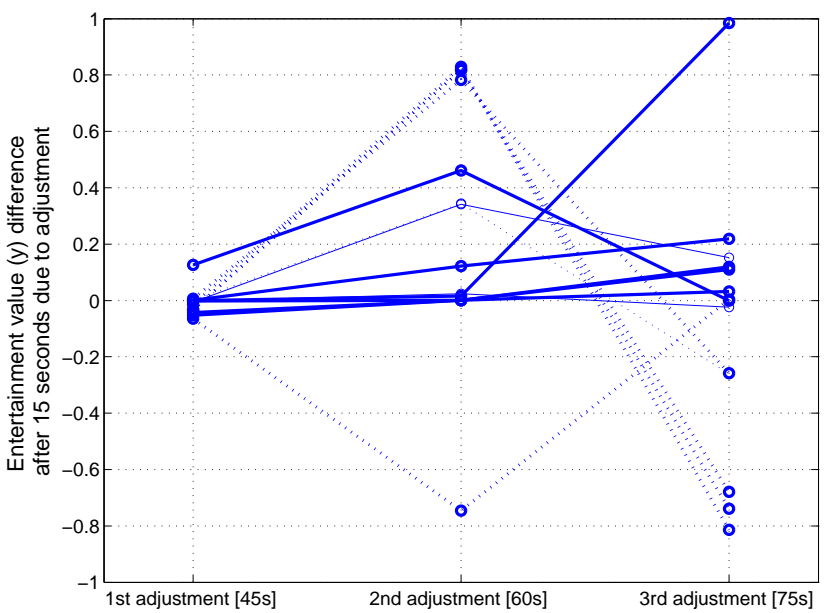

(a) The $18(A \succ S)$ instances

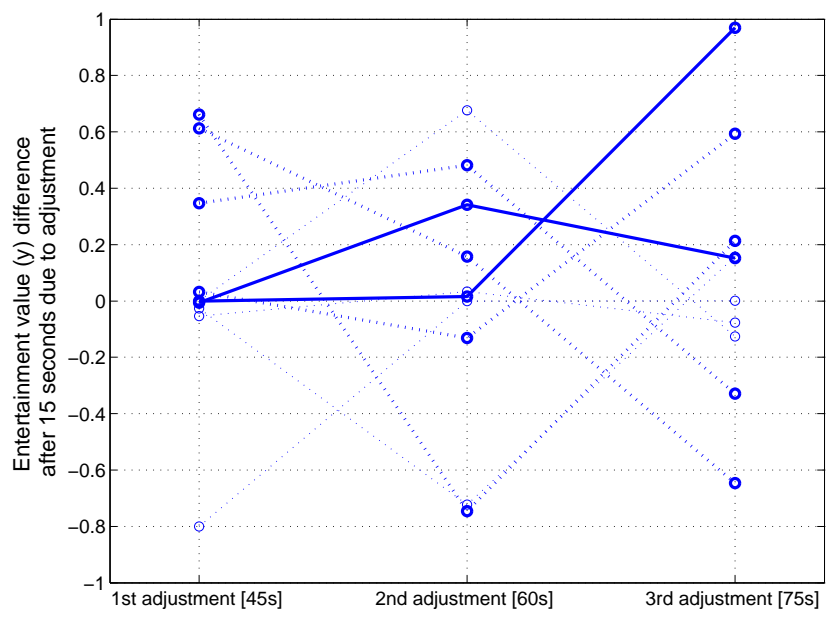

(b) The $12(A \prec S)$ instances

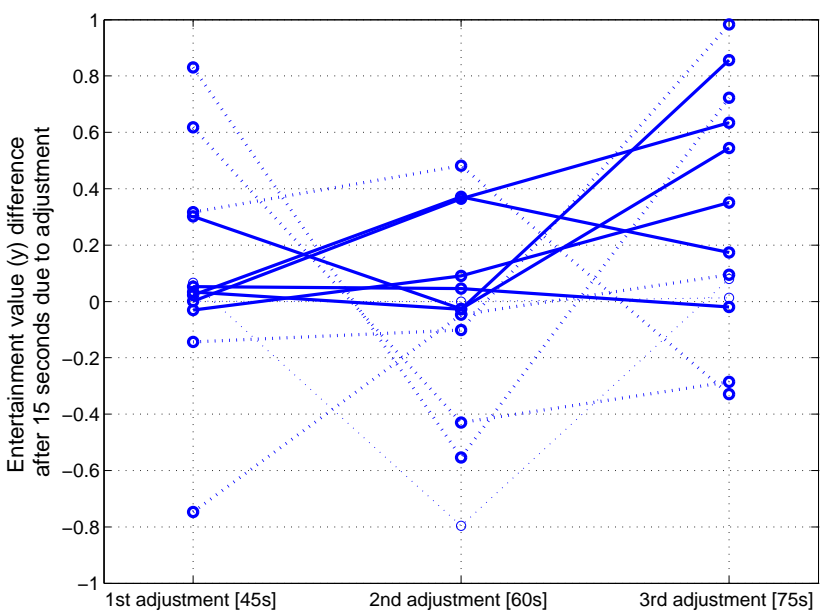

(c) The $20(A=S)$ instances

Fig. 4. $y$ difference values due to the game parameter adjustments occurring at the $45^{t h}, 60^{t h}$ and the $75^{t h}$ second of the adaptive game. The difference in the entertainment value is computed 15 seconds after each adjustment. Solid lines (bold or not) correspond to adaptive games free of maladjustments. Bold lines (solid or dotted) correspond to adaptive games that generate higher $y$ value than the respective $y$ value generated by the static game $\left(y_{a}>y_{s}\right)$. 
some indications about the level of 'fun' during the game as a non-intrusive alternative to questions. That strategy, however, implies a new assumption, that 'fun' is highly correlated to, or can easily be inferred from, sympathetic arousal.

Fun is almost certainly not constant during a game, but the performance of the adaptive controller suggests that this is not of vital importance, at least for Bug-Smasher. Additionally, the ANN entertainment preference models built on data derived from the game as a whole were evaluated on several smaller in-game time windows with the suggestive result that the difference between models' prediction accuracy on the whole 90 -second play window and their prediction accuracy on the 9 -second time window is approximately $16 \%$.

Another point of concern is that the generated model is the composite of subjective preferences of several subjects. The model is thus not perfectly adapted for any individual child playing the game. Results here and in our previous work [4] demonstrate that such composite models do nevertheless give good preference prediction performance. Models might be further individualized by on-line model learning during gameplay - though we have at present no suggestions for how this might be realized.

One might also question the constraint (or assumption) that the preferences expressed by the children must be consistent. This is necessary for a consistent model to exist, though a (perhaps poorly-performing) model can still be constructed when the assumption is false. The assumption was tested in [4] using order effect statistics; preferences do not appear strongly pairwise inconsistent by such measures. It is not clear that they are transitively consistent, though. Nevertheless, given the performance of the models reported in [4] this is evidently not a significant issue.

One of the limitations of the proposed entertainment modeling approach lies in the complexity of entertainment as a mental state. The generated $y$ value cannot be regarded as a mental affective state approximator but is rather a correlate of expressed children's entertainment preferences. Despite this, the $y$ value serves the purpose of this work well as far as entertainment modeling and optimization is concerned. Using the proposed entertainment augmentation scheme, knowledge of the direction (from the partial derivative) in which specific controllable features should be adjusted is available through the model; however, the magnitude of such an adjustment is not known a priori. Thus, applying gradient search with a fixed step in a given game feature may unexpectedly lead to lower values of entertainment. This is a fundamental limitation of the gradient-ascent adaptive mechanism, which appears here to cause more significant maladjustments of the curiosity $(H)$ than the challenge $(s)$ parameter. This problem might be resolved by injecting more controllable feature (challenge, curiosity) states in the search space or by introducing machine learning in real-time as proposed below.

Note that curiosity is a directly adjustable game parameter that also serves as input to the model. The gradient of $y$ with respect to such game parameters can be computed and serves as a sufficient basis for control. However, speed (challenge) is a Bug Smasher game parameter which is not a model input. Control of challenge is derived from an a priori relationship, coded in the set of rules in Table II, between challenge and the player features that are inputs to the model. While gradient ascent suggests what changes to the player features will lead to higher $y$ value, the game controller has no direct way to effect such changes, and control of challenge functions as an indirect method for positively influencing the player features.

The obvious modification to the current, simple, mechanism is to apply (for example) temporal difference reinforcement learning, most likely via classifier systems, to derive the relationship between desired player feature changes and the game control actions that actually effect them - i.e. to learn analogues of the designer-created rule-set used here. In such an approach, the game internal controls (challenge, curiosity) could be adjusted (within limits) and the effect on player satisfaction monitored using the entertainment model. The observed satisfaction changes would then act as reinforcement for the actor process adjusting the controls. To speed up learning, the reinforcement learning system could be seeded with the gradient information from the entertainment measure. The results of learning could also be saved so that experience with a given player accumulates: the game then adapts to the particular player over time.

Such a mechanism not only addresses the issue of how to achieve desired modifications to player features, which by their nature cannot be directly controlled, but also handles the quantization problem implicit in the gradient ascent mechanism: the adjustment is only valid in the limit and any finite adjustment of the game controls may be an 'over-adjustment' resulting in a decrease of $y$. The principal disadvantage of such a learning scheme is likely to be its (slow) rate of learning the player's characteristics.

The entertainment modeling approach presented here is general for the majority of action games created with Playware since the quantitative measures of challenge and curiosity are estimated through the generic features of speed and spatial diversity of the opponent on the game's surface. Thus, these or similar measures could be used to adjust player satisfaction in any future game development on the Playware tiles. However, each specific game possesses individual entertainment features that might need to be identified, quantified and added to the candidate feature set. Therefore, more games of the same and/or other genres must be tested to validate this hypothesis.

The generated ANN model is built using data derived from time, location and intensity of foot pressure events. Therefore, the model itself could potentially be used to capture player satisfaction in exertainment games that involve similar interaction, e.g. Dance Dance Revolution and Wii fit. However, further user studies would be required to validate this hypothesis.

While the entertainment model presented is not directly applicable to different modes of interaction (e.g., key strokes of a screen-based game), the player satisfaction modeling mechanism and the real-time adaptive technique could potentially scale up to complex 3D commercial-standard screen-based games. Earlier work has already shown that the experimental methodology of entertainment modeling is successful with both prey/predator screen-based games and Playware games [32].

A similar experimental approach can be followed for any 
computer game under investigation. First, factors of entertainment (e.g. challenge) need to be identified by the game designer. For each qualitative factor identified, one or more quantitative measures or variables available in the game and correlated with that factor need to be instrumented. The measures could typically include information on the game (e.g. speed, level complexity) and opponent behavior; these measures define the game feature set which can be used to generate a pool of dissimilar game variants (e.g. a slow vs. a fast game).

Features of playing behavior should also be identified and measured from the player's interactions with the game (e.g. time spent on a task, alternatives chosen, tracking of movement, use of items and contraptions). Meta-features combining two or more player features may also be relevant but could be automatically designed through the user modeling approach proposed. Human survey experiments are then devised to record game and player features and reported expressed emotions for the game variants designed ( $c f$. Section III). Complementary to 'fun', the game designer might choose additional emotions to investigate further: questionnaires exploring additional affective states, e.g. interestingness, excitement, frustration, boredom etc., could be designed. Note that comparative emotion analysis [62] using 4-AFC is strongly recommended for eliciting subjective reports of emotional preference or state.

Preference learning, through e.g. neuro-evolution, builds on the recorded player and game features to construct nonlinear mathematical expressions that correlate with or predict the player-reported emotions. Feature selection combined with preference learning can isolate those player and game features that contribute to predicting reported emotions, resulting in cognitive/affective models represented by ANNs. A game designer can then handcraft the whole playing experience for the player using information such as the gradient of the ANN model, as presented in this paper. For instance, the designer may decide that a good player experience should comprise high values of interestingness and frustration at a certain game level, followed by low values of boredom and high values of excitement at the next level. The proposed approach automates parts of the modern game development process, such as quality assurance and testing, while also providing an alternative and more accurate method for crafting player experience compared to the standard practices of difficulty scaling and rubber band artificial intelligence which are used extensively in game development.

\section{Conclusions}

This paper introduced an adaptive approach for augmenting player satisfaction in Playware games in real-time. The first step is to make use of the player satisfaction models derived from entertainment modeling processes to adjust Bug-Smasher game parameters on-line. Previous studies [4] on preference learning through the combination of neuro-evolution and feature selection generated ANN models able to predict — based on player features comprising children's average response time, the variance of the pressure they exert on the tiles and the number of interactions with the playground, together with the game feature of curiosity - the children's entertainment preferences with a 3-fold cross-validation accuracy of $77.77 \%$ (binomial-distributed $\mathrm{p}$-value $=\underline{\mathbf{0 . 0 0 1 9}}$ ). These models are used here as the basis of the adaptive game controller.

The mechanism presented here uses gradient ascent, on one such entertainment model, with respect to model inputs - the number of interactions, average response time and curiosity metric. For instance, if the partial derivative of the entertainment value (model output) with respect to curiosity (model input) is positive, one can increase the curiosity level control a little and expect a positive increment in player enjoyment. On-line adjustments of both the speed (challenge) and spatial diversity (curiosity) of the game opponents are controlled by simple rules, using the model derivatives, that are applied 45, 60 and 75 seconds into the 90 second game.

A survey experiment for evaluating the performance of the adaptation mechanism was designed in which 24 children were asked to compare the standard (static) versus the adaptive variant of the Bug-Smasher game. Results reveal a preference for the adaptive Bug-Smasher game. A further statistical analysis of the generated entertainment value demonstrates the positive impact of real-time adaptation for the improvement of the gameplay experience of Playware users. The adaptive mechanism introduced here augments the entertainment value of the game in the majority $(75.67 \%$; p-value $=\underline{\mathbf{0 . 0 0 1 2}})$ of the initial conditions investigated. In other words, the game adapts to the specific child by significantly increasing the entertainment value generated compared to the corresponding (baseline) static game played by the same child.

A deeper analysis of the adaptation mechanism and its effect on the generated entertainment value reveals that maladjustments of the curiosity and challenge levels occur that lead to lower entertainment values. Statistical analysis demonstrates a significant impact of those maladjustments in reducing children's expressed preference for the adaptation game. However, the statistically significant improvement of the entertainment (y) values when real-time adaptation occurs provides encouraging evidence that real-time optimization, based on entertainment preference models, of player satisfaction is possible even with a simple gradient ascent adaptive mechanism like that tested here.

\section{ACKNOWLEDGMENTS}

This work was supported in part by the Danish Research Agency, Ministry of Science, Technology and Innovation (project no: 274-05-0511). The authors thank Henrik Jørgensen and all children of Henriette Hørlücks School, Odense, Denmark that participated in the experiments.

The authors also thank Alex Nareyek for insightful discussions.

The tiles were designed by C. Isaksen from Isaksen Design and parts of their hardware and software implementation were collectively done by A. Derakhshan, F. Hammer, T. Klitbo and J. Nielsen. KOMPAN, Mads Clausen Institute, and Danfoss Universe also participated in the development of the tiles. 


\section{REFERENCES}

[1] G. N. Yannakakis and J. Hallam, "Real-time Adaptation of AugmentedReality Games for Optimizing Player Satisfaction," in Proceedings of the IEEE Symposium on Computational Intelligence and Games. Perth, Australia: IEEE, December 2008, pp. 103-110.

[2] H. H. Lund, T. Klitbo, and C. Jessen, "Playware technology for physically activating play," Artifical Life and Robotics Journal, vol. 9, no. 4 , pp. $165-174,2005$.

[3] G. N. Yannakakis, H. H. Lund, and J. Hallam, "Modeling Children's Entertainment in the Playware Playground," in Proceedings of the IEEE Symposium on Computational Intelligence and Games. Reno, USA: IEEE, May 2006, pp. 134-141.

[4] G. N. Yannakakis and J. Hallam, "Game and Player Feature Selection for Entertainment Capture," in Proceedings of the IEEE Symposium on Computational Intelligence and Games. Hawaii, USA: IEEE, April 2007, pp. 244-251.

[5] T. W. Malone, "What makes computer games fun?" Byte, vol. 6, pp. 258-277, 1981.

[6] G. N. Yannakakis, M. Maragoudakis, and J. Hallam, "Preference Learning for Cognitive Modeling: A Case Study on Entertainment Preferences," IEEE Systems, Man and Cybernetics; Part A: Systems and Humans, 2008, (to appear).

[7] "IEEE Task Force on Player Satisfaction Modeling," IEEE Computational Intelligence Society, 2007, available at http://game.itu.dk/PSM/.

[8] M. Csikszentmihalyi, Beyond Boredom and Anxiety: Experiencing Flow in Work and Play. San Francisco: Jossey-Bass, 2000.

[9] _ Flow: The Psychology of Optimal Experience. New York: Harper \& Row, 1990

[10] P. Sweetser and P. Wyeth, "GameFlow: A Model for Evaluating Player Enjoyment in Games," ACM Computers in Entertainment, vol. 3, no. 3, July 2005.

[11] B. Cowley, D. Charles, M. Black, and R. Hickey, "Toward an Understanding of Flow in Video Games," ACM Computers in Entertainment, vol. 6 , no. 2, July 2008 .

[12] N. Lazzaro, "Why we play games: Four keys to more emotion without story," in Game Developers Conference, 2004.

[13] R. Koster, A Theory of Fun for Game Design. Paraglyph Press, 2005.

[14] C. Bateman and R. Boon, 21st Century Game Design. Charles River Media, 2005.

[15] J. Read, S. MacFarlane, and C. Cassey, "Endurability, engagement and expectations," in Proceedings of International Conference for Interaction Design and Children, 2002.

[16] R. J. Pagulayan, K. Keeker, D. Wixon, R. L. Romero, and T. Fuller, The Human-Computer Interaction Handbook: Fundamentals, Evolving Technologies and Emerging Applications. NJ: Lawrence Erlbaum Associates, 2003, ch. User-centered design in games, pp. 883-906.

[17] R. J. Pagulayan and K. Keeker, Handbook of Formal and Informal Interaction Design Methods. San Francisco: Morgan Kaufmann Publishers, 2007, ch. Measuring Pleasure and Fun: Playtesting.

[18] W. A. Ijsselsteijn, Y. A. W. de Kort, K. Poels, A. Jurgelionis, and F. Belotti, "Characterising and measuring user experiences," in $A C E$ 2007 International Conference on Advances in Computer Entertainment Technology, 2007.

[19] G. Calleja, "Digital games as designed experience: Reframing the concept of immersion," Ph.D. dissertation, Victoria University of Wellington, New Zealand, 2007.

[20] R. M. Ryan, C. S. Rigby, and A. Przybylski, "The motivational pull of video games: A self-determination theory approach," Motivation and Emotion, vol. 30, no. 4, pp. 344-360, 2006.

[21] R. Likert, "A technique for the measurement of attitudes," Archives of Psychology, vol. 140, pp. 1-55, 1932.

[22] H. Iida, N. Takeshita, and J. Yoshimura, "A metric for entertainment of boardgames: its implication for evolution of chess variants," in IWEC2002 Proceedings, R. Nakatsu and J. Hoshino, Eds. Kluwer, 2003, pp. 65-72.

[23] G. van Lankveld, P. Spronck, and M. Rauterberg, "Difficulty Scaling through Incongruity," in Proceedings of the 4th International Artificial Intelligence and Interactive Digital Entertainment Conference. AAAI Press, 2008, pp. 228-229.

[24] M. Rauterberg, "About a framework for information and information processing of learning systems," in Information System Concepts. IFIP Chapman \& Hall, 1995, pp. 54-69.

[25] N. Lazzaro, "Why we play games: Four keys to more emotion without story," XEO Design Inc., Technical Report, 2004.
[26] G. N. Yannakakis and J. Hallam, "Evolving Opponents for Interesting Interactive Computer Games," in From Animals to Animats 8: Proceedings of the $8^{\text {th }}$ International Conference on Simulation of Adaptive Behavior (SAB-04), S. Schaal, A. Ijspeert, A. Billard, S. Vijayakumar, J. Hallam, and J.-A. Meyer, Eds. Santa Monica, LA, CA: The MIT Press, July 2004, pp. 499-508.

[27] — "Towards Optimizing Entertainment in Computer Games," Applied Artificial Intelligence, vol. 21, pp. 933-971, 2007.

[28] — "A Generic Approach for Obtaining Higher Entertainment in Predator/Prey Computer Games," Journal of Game Development, vol. 1, no. 3, pp. 23-50, December 2005.

[29] N. Beume, H. Danielsiek, C. Eichhorn, B. Naujoks, M. Preuss, K. Stiller, and S. Wessing, "Measuring Flow as Concept for Detecting Game Fun in the Pac-Man Game," in Proc. 2008 Congress on Evolutionary Computation (CEC'08) within Fifth IEEE World Congress on Computational Intelligence (WCCI'08). IEEE, 2008, pp. 3447-3454.

[30] N. Beume, T. Hein, B. Naujoks, G. Neugebauer, N. Piatkowski, M. Preuss, R. Stoer, and A. Thom, "To Model or Not to Model: Controlling Pac-Man Ghosts Without Incorporating Global Knowledge," in Proc. 2008 Congress on Evolutionary Computation (CEC'08) within Fifth IEEE World Congress on Computational Intelligence (WCCI'08). IEEE, 2008, pp. 3463-3470.

[31] G. N. Yannakakis and J. Hallam, "Towards Capturing and Enhancing Entertainment in Computer Games," in Proceedings of the $4^{\text {th }}$ Hellenic Conference on Artificial Intelligence, Lecture Notes in Artificial Intelligence, vol. 3955. Heraklion, Greece: Springer-Verlag, May 2006, pp. $432-442$.

[32] _ - "Modeling and augmenting game entertainment through challenge and curiosity," International Journal on Artificial Intelligence Tools, vol. 16, no. 6, pp. 981-999, December 2007.

[33] R. L. Mandryk, K. M. Inkpen, and T. W. Calvert, "Using Psychophysiological Techniques to Measure User Experience with Entertainment Technologies," Behaviour and Information Technology (Special Issue on User Experience), vol. 25, no. 2, pp. 141-158, 2006.

[34] R. L. Mandryk and M. S. Atkins, "A Fuzzy Physiological Approach for Continuously Modeling Emotion During Interaction with Play Environments," International Journal of Human-Computer Studies, vol. 65, pp. 329-347, 2007.

[35] N. Ravaja, T. Saari, M. Turpeinen, J. Laarni, M. Salminen, and M. Kivikangas, "Spatial Presence and Emotions during Video Game Playing: Does It Matter with Whom You Play?" Presence Teleoperators \& Virtual Environments, vol. 15, no. 4, pp. 381-392, 2006.

[36] R. L. Hazlett, "Measuring emotional valence during interactive experiences: boys at video game play," in CHI '06: Proceedings of the SIGCHI conference on Human Factors in computing systems. New York, NY, USA: ACM Press, 2006, pp. 1023-1026.

[37] P. Rani, N. Sarkar, and C. Liu, "Maintaining optimal challenge in computer games through real-time physiological feedback," in Proceedings of the $11^{\text {th }}$ International Conference on Human Computer Interaction, 2005.

[38] J. Sinclair, P. Hingston, and M. Masek, "Considerations for the design of exergames," in GRAPHITE '07: Proceedings of the 5th international conference on Computer graphics and interactive techniques in Australia and Southeast Asia. ACM, 2007, pp. 289-295.

[39] G. Andrade, G. Ramalho, H. Santana, and V. Corruble, "Extending reinforcement learning to provide dynamic game balancing," in Proceedings of the Workshop on Reasoning, Representation, and Learning in Computer Games, 19th International Joint Conference on Artificial Intelligence (IJCAI), August 2005, pp. 7-12.

[40] M. A. Verma and P. W. McOwan, "An adaptive methodology for synthesising Mobile Phone Games using Genetic Algorithms," in Congress on Evolutionary Computation (CEC-05), Edinburgh, UK, September 2005, pp. 528-535.

[41] R. Hunicke and V. Chapman, "AI for Dynamic Difficulty Adjustment in Games," in Proceedings of the Challenges in Game AI Workshop, $19^{\text {th }}$ Nineteenth National Conference on Artificial Intelligence (AAAI'04), 2004.

[42] P. Spronck, I. Sprinkhuizen-Kuyper, and E. Postma, "Difficulty Scaling of Game AI," in Proceedings of the 5th International Conference on Intelligent Games and Simulation (GAME-ON 2004), 2004, pp. 33-37.

[43] J. Ludwig and A. Farley, "A learning infrastructure for improving agent performance and game balance," in Proceedings of the AIIDE'07 Workshop on Optimizing Player Satisfaction, Technical Report WS-0701, G. N. Yannakakis and J. Hallam, Eds. AAAI Press, 2007, pp. $7-12$.

[44] J. K. Olesen, G. N. Yannakakis, and J. Hallam, "Real-time challenge balance in an RTS game using rtNEAT," in Proceedings of the IEEE 
Symposium on Computational Intelligence and Games. Perth, Australia: IEEE, December 2008, pp. 87-94.

[45] B. D. Bryant and R. Miikkulainen, "Acquiring Visibly Intelligent Behavior with Example-Guided Neuroevolution," in Proceedings of the Twenty-Second National Conference on Artificial Intelligence (AAAI-07). AAAI Press, 2007, pp. 801-808.

[46] H. Barber and D. Kudenko, "A user model for the generation of dilemma-based interactive narratives," in Proceedings of the AIIDE'07 Workshop on Optimizing Player Satisfaction, Technical Report WS-0701, G. N. Yannakakis and J. Hallam, Eds. AAAI Press, 2007, pp. $13-18$.

[47] D. L. Roberts, C. R. Strong, and C. L. Isbell, "Estimating player satisfaction through the author's eyes," in Proceedings of the AIIDE'07 Workshop on Optimizing Player Satisfaction, Technical Report WS-0701, G. N. Yannakakis and J. Hallam, Eds. AAAI Press, 2007, pp. $31-36$.

[48] D. Thue, V. Bulitko, M. Spetch, and E. Wasylishen, "Learning player preferences to inform delayed authoring," in Papers from the AAAI'07 Fall Symposium on Intelligent Narrative Technologies. AAAI Press, 2007.

[49] J. Togelius, R. D. Nardi, and S. M. Lucas, "Making racing fun through player modeling and track evolution," in Proceedings of the SAB Workshop on Adaptive Approaches to Optimizing Player Satisfaction, G. N. Yannakakis and J. Hallam, Eds., Rome, 2006, pp. 61-70.

[50] — - "Towards automatic personalised content creation for racing games," in Proceedings of the IEEE Symposium on Computational Intelligence and Games. Hawaii, USA: IEEE, April 2007, pp. 252-259.

[51] G. N. Yannakakis and J. Hallam, "A generic approach for generating interesting interactive pac-man opponents," in Proceedings of the IEEE Symposium on Computational Intelligence and Games, G. Kendall and S. M. Lucas, Eds., Essex University, Colchester, UK, 4-6 April 2005, pp. 94-101.

[52] - "A scheme for creating digital entertainment with substance," in Proceedings of the Workshop on Reasoning, Representation, and Learning in Computer Games, 19th International Joint Conference on Artificial Intelligence (IJCAI), August 2005, pp. 119-124.

[53] G. N. Yannakakis, J. Levine, and J. Hallam, "An Evolutionary Approach for Interactive Computer Games," in Proceedings of the Congress on Evolutionary Computation (CEC-04), June 2004, pp. 986-993.

[54] G. N. Yannakakis and M. Maragoudakis, "Player modeling impact on player's entertainment in computer games," in Proceedings of the $10^{\text {th }}$ International Conference on User Modeling; Lecture Notes in Computer Science, vol. 3538. Edinburgh: Springer-Verlag, 24-30 July 2005, pp. 74-78.

[55] F. Hammer, A. Derakhshan, and H. H. Lund, "Adapting Playgrounds for Children Play using Ambient Playware," in Proceedings of the 2006 IEEE/RSJ International Conference on Intelligent Robots and Systems (IROSO6), Beijing, China, October 9-15 2006, pp. 5625-5630.

[56] G. N. Yannakakis, "AI in Computer Games: Generating Interesting Interactive Opponents by the use of Evolutionary Computation," Ph.D. thesis, University of Edinburgh, November 2005.

[57] J. Togelius and J. Schmidhuber, "An Experiment in Automatic Game Design," in Proceedings of the IEEE Symposium on Computational Intelligence and Games. Perth, Australia: IEEE, December 2008, pp. 252-259.

[58] P. Devijver and J. Kittler, Pattern Recognition - A Statistical Approach. Engelwood cliffs, NJ: Prentice-Hall, 1982.

[59] J. Doyle, "Prospects for preferences," Computational Intelligence, vol. 20, no. 2, pp. 111-136, May 2004.

[60] G. N. Yannakakis, J. Hallam, and H. H. Lund, "Entertainment Capture through Heart Rate Activity in Physical Interactive Playgrounds," User Modeling and User-Adapted Interaction, Special Issue: Affective Modeling and Adaptation, vol. 18, no. 1-2, pp. 207-243, February 2008.

[61] R. W. Picard, E. Vyzas, and J. Healey, "Toward Machine Emotional Intelligence: Analysis of Affective Physiological State," IEEE Trans. Pattern Anal. Mach. Intell., vol. 23, no. 10, pp. 1175-1191, 2001.

[62] G. N. Yannakakis, J. Hallam, and H. H. Lund, "Comparative Fun Analysis in the Innovative Playware Game Platform," in Proceedings of the $1^{\text {st }}$ World Conference for Fun 'n Games, 2006, pp. 64-70.

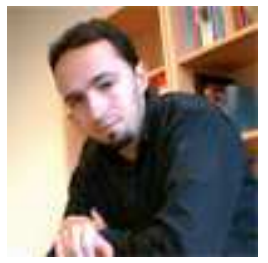

Georgios N. Yannakakis (Member, IEEE) is Associate Professor at the IT University of Copenhagen. He received both the 5-year Diploma (1999) in Production Engineering and Management and the M.Sc. (2001) degree in Financial Engineering from the Technical University of Crete and the Ph.D. degree in Informatics from the University of Edinburgh in 2005. Prior to joining the Center for Computer Games Research, IT University of Copenhagen in 2007, he was a postdoctoral researcher at the Maersk

Denmark

Mc-Kinney Moller Institute, University of Southern

His research interests include computational intelligence in games, user (cognitive and affective) modeling, artificial life, neuro-evolution and emergent cooperation within multi-agent systems. He has published around 40 journal and international conference papers on the aforementioned fields. He is the chair of the IEEE Task Force on Player Satisfaction Modeling.

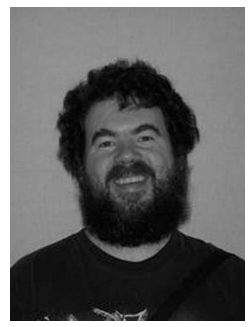

John Hallam graduated with First Class Honors in Mathematics from the University of Oxford in 1979, completed a Ph.D. in the Department of Artificial Intelligence at the University of Edinburgh in 1984 and joined the teaching Faculty in that Department in 1985. He established the Edinburgh Mobile Robotics Research Group, having been active in mobile robotics research for almost 20 years. In 2003 he moved to the Mærsk Institute at the University of Southern Denmark.

The current focus of his catholic research interest is in biological modeling using robotic techniques, evolutionary robotics, collective robotics and modeling of user satisfaction in computer games. He has published around 100 journal and international conference papers on various robotic and non-symbolic computing topics, and has designed electronic hardware both for research and teaching and commercially. $\mathrm{He}$ is President of the International Society for Adaptive Behaviour, a member of the London Mathematical Society, and a Director of 3 Lions Design Ltd., a small company that does commercial electronic design. 